\title{
Independent validation of Swarm Level 2 magnetic field products and 'Quick Look' for Level 1b data
}

\author{
Ciarán D. Beggan, Susan Macmillan, Brian Hamilton, and Alan W. P. Thomson
}

British Geological Survey, Murchison House, Edinburgh, EH9 3LA U.K.

(Received December 19, 2012; Revised August 14, 2013; Accepted August 21, 2013; Online published November 22, 2013)

\begin{abstract}
Magnetic field models are produced on behalf of the European Space Agency (ESA) by an independent scientific consortium known as the Swarm Satellite Constellation Application and Research Facility (SCARF), through the Level 2 Processor (L2PS). The consortium primarily produces magnetic field models for the core, lithosphere, ionosphere and magnetosphere. Typically, for each magnetic product, two magnetic field models are produced in separate chains using complementary data selection and processing techniques. Hence, the magnetic field models from the complementary processing chains will be similar but not identical. The final step in the overall L2PS therefore involves inspection and validation of the magnetic field models against each other and against data from (semi-) independent sources (e.g. ground observatories). We describe the validation steps for each magnetic field product and the comparison against independent datasets, and we show examples of the output of the validation. In addition, the L2PS also produces a daily set of 'Quick Look' output graphics and statistics to monitor the overall quality of Level $1 \mathrm{~b}$ data issued by ESA. We describe the outputs of the 'Quick Look' chain.
\end{abstract}

Key words: Swarm, validation, quick look, magnetic modelling.

\section{Introduction}

\subsection{Overview}

Swarm is a European Space Agency (ESA) mission consisting of three low Earth orbiting satellites designed specifically to measure magnetic and electric fields to a greater accuracy than ever before (Friis-Christensen et al., 2006). Two lower satellites fly at a $450 \mathrm{~km}$ nominal altitude in nearidentical orbits, separated by 15 seconds in time at their equatorial crossing, while the third flies in a higher orbit at about $530 \mathrm{~km}$. The orbits of the two lower satellites drift relative to the upper satellite over the course of the nominal mission (four years), gradually achieving local time separation to improve the temporal sensitivity of the data, while the lower two satellites will provide improved spatial sensitivity through the use of gradient information. Note the altitude of the three satellites will decrease over the mission lifetime.

All three satellites have identical instrumentation. The primary instruments for sensing the magnetic field are (a) a vector magnetometer on a fixed optical bench with three star cameras and (b) a low-noise scalar magnetometer, located at the end of a long boom to shield it from stray magnetic fields. The star cameras are used to determine the orientation of the vector instrument and hence the field in space while the scalar magnetometer provides a steady and reliable reference point for the vector magnetometer. The electric field is sensed using a novel ion sensor and Langmuir probes. The satellites have two on-board Global Positioning Satellite systems, which in conjunction with post-

Copyright (C) NERC, 2013. All Rights Reserved.

doi:10.5047/eps.2013.08.004 processing of the orbital data using gravity field models will produce a precise orbit determination accurate to centimetres (Visser et al., 2013).

The raw satellite measurements are processed by ESA (e.g. transformed from engineering units and rotated to the geocentric reference frame) to produce the so-called 'Level $1 \mathrm{~b}$ ' data which represents the best estimate of the actual values of the electric and magnetic field at each point in space and time. The Level $1 \mathrm{~b}$ products are released by ESA and are available via the internet to the scientific community.

In addition to the Level $1 \mathrm{~b}$ data release, ESA has commissioned a consortium of scientific experts to produce the so-called 'Level 2' magnetic and electric field models and products to act as standards for the general scientific community. The Swarm Satellite Constellation Application and Research Facility (SCARF) is a consortium of six European institutes with acknowledged capability and expertise in magnetic and electric field modelling and orbit determination (Olsen et al., 2013). The SCARF will operate the Level 2 Processing System (L2PS) to provide the Level 2 products to the user community.

Level 2 products consist of a series of scientifically accurate magnetic field, electric field, precise orbit determination and upper atmospheric wind models, which use Level $1 \mathrm{~b}$ data (and auxiliary sources) for their computation. For example, the four main components of the magnetic field measurable in low Earth orbit are the (1) core, (2) lithospheric, (3) ionospheric and (4) magnetospheric fields. Each of these sources have distinctive temporal and spatial characteristics which allow them to be separated from each other given enough suitable data. In addition, magnetic and 


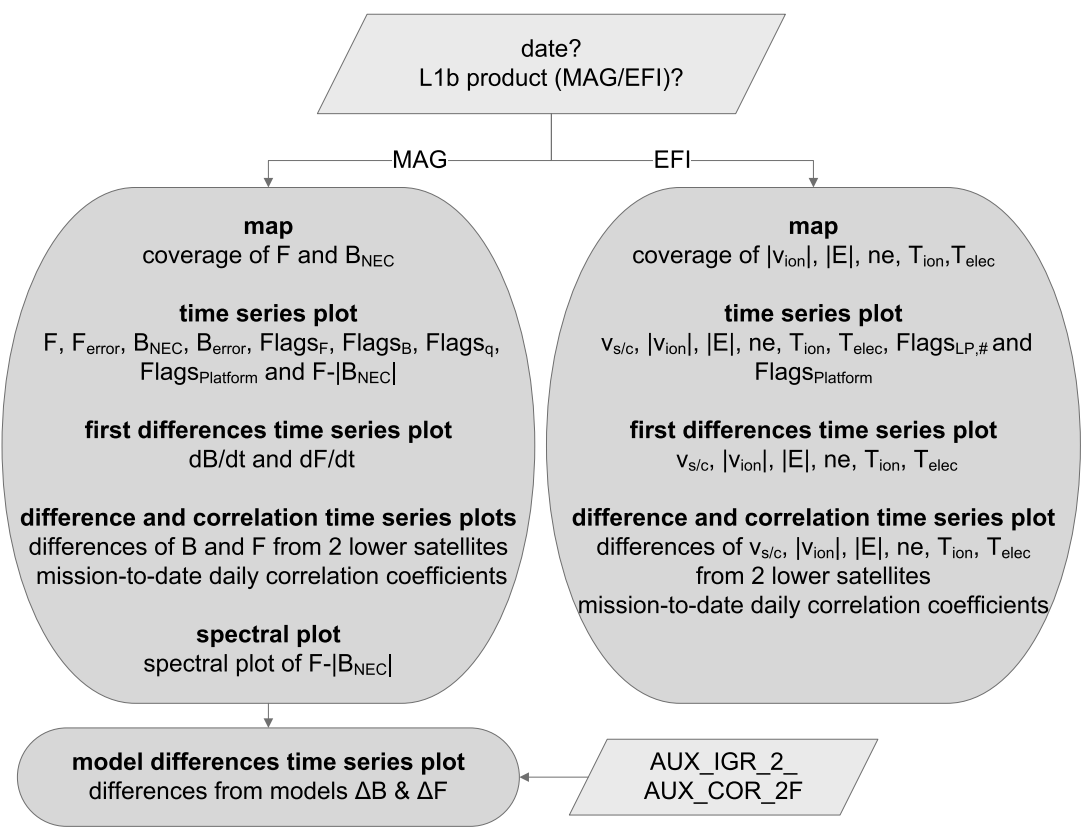

Fig. 1. Work flow diagram for Quick-Look chain. Rhomboids indicate input data. Round-cornered rectangles are the output data from the processing algorithms. MAG refers to the magnetic field products; EFI refers to the Electric field products. See Section 2 for details about the graphs and maps plotted.

electric fields generated by other geophysical sources will also be investigated as part of the Swarm mission; for example, the mission should give insights into the flow of ocean currents, mantle conductivity at depth and descriptions of other poorly understood phenomena such as the equatorial electrojet. We will briefly describe the input data and some of the output products in the paper.

The consortium will also run a 'Quick Look' processing chain, designed to produce daily plots and mission-to-date analyses of the Level $1 \mathrm{~b}$ data. This chain will examine the outputs to check for data consistency and quality, thus acting as a secondary source of scientific expertise for ESA.

In Section 2, we outline the daily processing tasks and tests carried out on the Level 1b data in the 'Quick Look' chain for the L2PS. In the Section 3 we describe, briefly, the generation of Level 2 products and the validation tests carried out. We show some example results from a synthetic test dataset used to produce and evaluate the L2PS operation and performance.

\section{2. 'Quick Look' of Level 1b Data}

The Level 2 Quick-Look (QL) chain uses Swarm Level $1 \mathrm{~b}$ magnetic (MAG) $(1 \mathrm{~Hz})$ and plasma/electric field (EFI) $(2 \mathrm{~Hz})$ products as input (specifically the products MAG $X \_L R \_1 B$ and EFIX_PL_1B where $X$ is satellite A, B or $\mathrm{C}$ ) and outputs daily quick-look reports in Adobe PDF format. These reports include daily and mission-to-date plots and their main purpose is to provide simple graphical representations of the Swarm Level $1 b$ data to monitor their overall quality. They are intended to complement, not duplicate, the quality assessments within the ESA Level $1 \mathrm{~b}$ processing system. The work flow diagram for the QL chain in Fig. 1 shows the inputs to the process (rhomboids) and the output plots, statistics and data (round-cornered rectangles) from the processing algorithms.
For the Level 1b magnetic field data, the total field strength $(F)$ and the strength of the North, East and Centre $\left(B_{\mathrm{NEC}}\right)$ components of the magnetic field are drawn as spatial maps to aid identification of spatial patterns or gaps. Time series plots of the measured $F$ and $B_{\mathrm{NEC}}$ values and the closure error (the absolute difference $\left(F-\left|B_{\mathrm{NEC}}\right|\right)$, as well as associated error flags and first derivatives $(d B / d t$ and $d F / d t$ ) are generated to check for gross errors, steps or spikes. The spectra of differences between $F$ and $\left|B_{\mathrm{NEC}}\right|$ and the correlation of data between satellites will be useful for examining periodic signals or identifying changes over time between them.

For the electric field Level $1 \mathrm{~b}$ data, the spatial coverage of the ion drift velocity $\left(v_{\text {ion }}\right)$, the total electric field strength $(|E|)$, the total electron count $\left(n_{e}\right)$, and the temperature of the ions and electrons ( $T_{\mathrm{ion}}$ and $T_{e}$ ) will be mapped. Time series plots of these values and the velocity of the spacecraft $\left(v_{s / c}\right)$ will be generated to look for abnormal spikes or steps in the data, as well as to examine the relevant error flags. Other plots (e.g. monthly temporal correlation of each satellite dataset) and statistics (e.g. data availability) will also be computed. The daily Quick-Look products will be available to the general community.

The purpose of each plot for the magnetic data, in terms of quick-look and basic quality control, is listed in Table 1. The QL chain has been tested with synthetic data (called TDS-1, see Section 4) and an example of a time series plot of differences is shown in Fig. 2.

\section{Production of Level 2 Products from Level $1 \mathrm{~b}$ Data}

The Level $1 \mathrm{~b}$ data are produced by ESA and delivered in the form of single day files for each satellite. Each magnetic field day file consists of the geocentric position (i.e. latitude, longitude and radius) of the satellite along with the relevant 
Table 1. Daily Quick-Look images and purpose for magnetic field Level $1 \mathrm{~b}$ data. See Section 2 for a detailed explanation.

\begin{tabular}{|l|l|}
\hline Quick Look graphical image & Purpose \\
\hline Map showing coverage of $F$ and $B_{\mathrm{NEC}}$ from each satellite & Identification of spatially correlated data gaps \\
\hline $\begin{array}{l}\text { Time series plots: differences between } F \text { (total field strength) } \\
\text { and }\left|B_{\mathrm{NEC}}\right| \text { (strength of the North, East and Centre components) }\end{array}$ & Identification of gross errors and inspection of flag values \\
\hline Time series plots: first differences for each satellite & Identification of steps and spikes \\
\hline $\begin{array}{l}\text { Inter-satellite (lower pair) differences, time series plots, } \\
\text { daily correlations for mission to date }\end{array}$ & Identification of steps and spikes in data \\
\hline $\begin{array}{l}\text { Spectra of differences between } F \text { and }\left|B_{\mathrm{NEC}}\right| \\
\text { Time series plot: differences from model }\end{array}$ & For detection of periodic signals \\
\hline
\end{tabular}

Differences between TDS-1 satellite A magnetic data for 02-Jul-1998 and auxiliary model

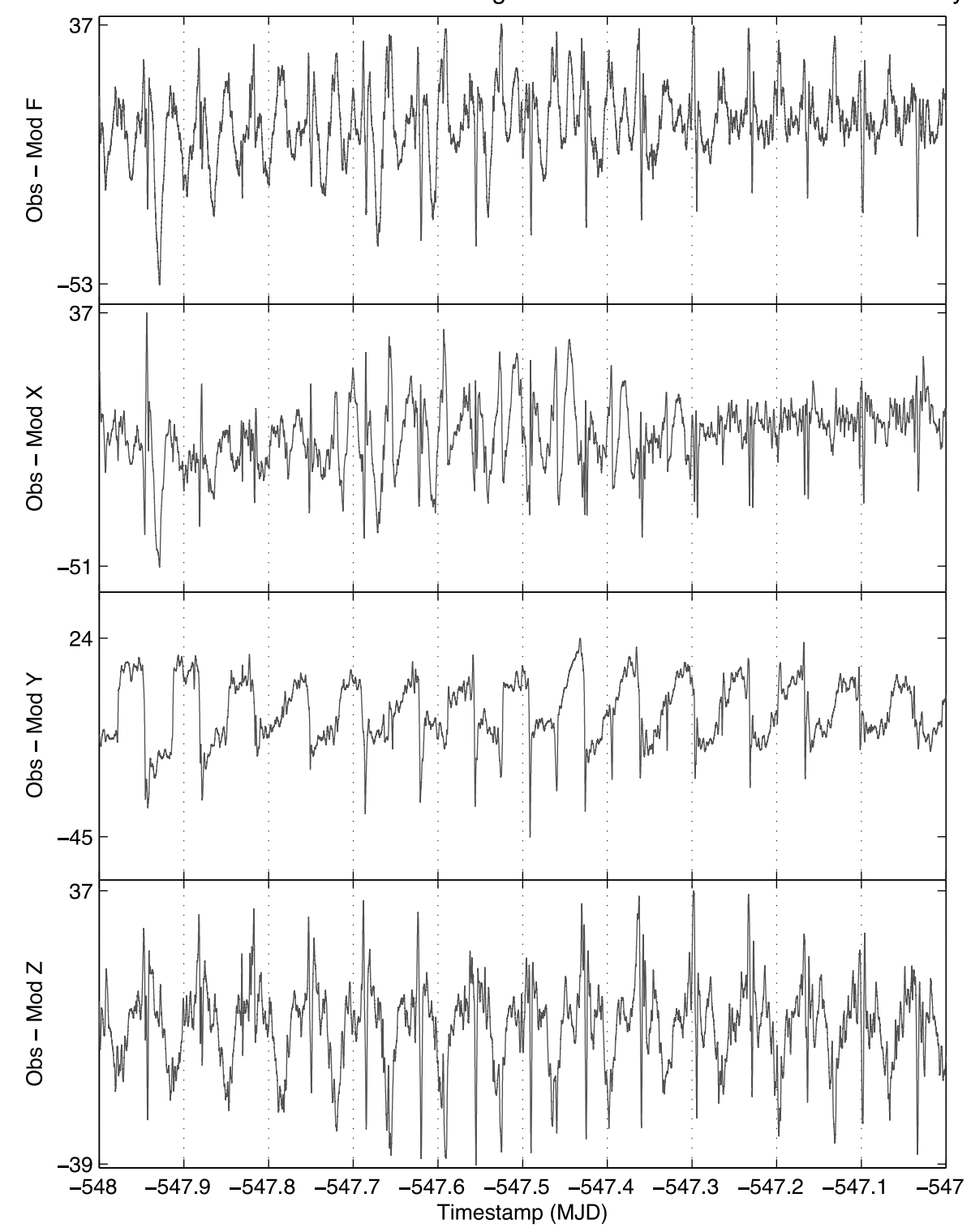

Fig. 2. Example of a Quick-Look daily image for magnetic data for Modified Julian Day (MJD) 519 showing the differences between the observed (Obs) magnetic field from Swarm A and the values predicted from an auxiliary core field model (Mod) for the North $(X)$, East $(Y)$ and Down $(Z)$ components and Total Field $(F)$ values. Units: nT.

data; for example, a magnetic field day file contains the measured values for each second of the three orthogonal components (i.e. $B_{r}, B_{\theta}, B_{\phi}$ ) and total scalar field values, along with various data quality flag indicators (e.g. missing data; star camera availability; spacecraft manoeuvres).
Under the direction of ESA, SCARF members produce one or more of the magnetic or electric field models. Level 1b data is ingested by the SCARF institutes and processed into the various Level 2 products. The schedule of product release varies depending on the model. For exam- 
ple, there will be a fast track core field model released every quarter, but the dedicated core field model will only be released after first six months and, thereafter, annually. As the Swarm mission progresses, larger datasets will allow the different products to be refined. The lithospheric and ionospheric models will improve as longer and larger datasets become available. In turn better models of the core can be produced, hence improving the other models in the next iteration of product releases.

There are three types of Level 2 products: Category 1 (CAT-1), Category 1 fast-track and Category 2 (CAT-2) products. CAT-1 products include magnetic field models produced by SCARF which require expert knowledge (e.g. for data selection) and take weeks to months in processing time to elicit the best model. CAT-1 fast-track products are produced relatively rapidly by $\mathrm{L} 2 \mathrm{PS}$, for example the fasttrack magnetospheric model is released on a daily basis, while the fast-track core field model is released quarterly. In contrast, CAT-2 products are computed automatically from the Level $1 \mathrm{~b}$ data in less than a few hours without expert intervention and hence will be produced by ESA soon after the release of the Level $1 \mathrm{~b}$ data.

The Level 2 CAT-1 magnetic field models are produced in two general streams. The first processing chain uses a Comprehensive Inversion (CI) approach (Sabaka and Olsen, 2006; Sabaka et al., 2013) which attempts to invert the entire magnetic dataset for all modelled field constituents simultaneously. Thus, the CI models of the core, lithosphere, ionosphere and magnetosphere are produced simultaneously. Another set of field models are computed by individual dedicated chains. For example, the dedicated core field model chain will solve primarily for the core field from night-side only data (though it also co-estimates models of the lithosphere and magnetosphere) (Rother et al., 2013). Each of the products is self-validated by the institute producing it, before intermediary release to ESA and the other SCARF members. All products are accompanied by an intermediate validation report.

The product names adhere to strict rules and are of fixed length. The file name consists of the first two letters SW for Swarm, followed by an underscore. The next four letters are OPER indicating it is operational data. The detailed product type is given in the next ten letters such as MCO_SHA_2D, indicating the Magnetic field COre model Spherical Harmonic Analysis coefficients (Level 2 product) from the Dedicated processing chain. Finally, the validity dates of the model are given along with a version number consisting of four numerals. For example, a release of version 1 of a Dedicated core field model valid from midnight on 01 June 2013 to midnight 01 June 2014 would have the following file name: SW_OPER_MCO_SHA_2D_20130601T000000_ 20140601T000000_0001.

Before a Level 2 CAT-1 product file is released to the general community, it undergoes a number of checks carried out by an independent partner within L2PS. The product initially has an intermediate name with an additional ' $i$ ' in the filename (e.g., SW_OPER_MCO_SHAi2D_ 20130601T000000_20140601T000000_0001) and is released to other members of the SCARF. The intermediate product is checked by a knowledgeable scientist for er- rors and/or subtle differences between it, its complementary product from another chain (if available) and other data or other models produced independently of the Swarm mission (where applicable).

When the model has been independently checked and validated by L2PS, the ' $\mathrm{i}$ ' is removed (e.g., $\quad$ SW_OPER_MCO_SHA_2D_ 20130601T000000_20140601T000000_0001) and the product is issued with a validation report (SW_OPER_MCO_ VAL_2D_20130601T000000_20140601T000000_0001) in which the results of the tests are given. The intermediary validation report from the institute which produced the product is also attached to the final validation report and L2PS pass their recommendation about the product to the ESA quality working group, who make the final decision to release the validated product.

Only CAT-1 models will be independently validated. The CAT-1 fast track models (core and magnetosphere) are produced quickly in order to be useful to various scientific communities who require rapid (but not necessarily completely accurate) models of these fields. As the CAT- 2 product chains are automatically produced on a daily basis, these products are not validated by L2PS either, though they are subject to scientific scrutiny and quality control checks by ESA quality working group. There are 15 CAT-1 products which will be validated by L2PS, as noted in Table 2 .

\section{Validation of Level 2 Products}

The SCARF consortium used a common reference Test Data Set for CAT-1 (TDS-1) for the purpose of development and testing of the Level 2 processing chain algorithms. The TDS-1 was constructed from spherical harmonic models of the core, lithosphere, ionosphere and magnetosphere and employed modelled orbital position data to construct a synthetic dataset at satellite altitudes. The dataset used a nominal mission lifetime of four-and-a-half years, starting from July 1998 until December 2003. The models were upward continued to Swarm satellite altitudes, the summed contributions from each field component were computed at the modelled orbital positions and then Gaussian noise was added to produce the common Level $1 \mathrm{~b}$ dataset. The synthetic Level $1 \mathrm{~b}$ data were then used to compute the Level 2 magnetic field reference models. The TDS-1 dataset was used as a reference to compare the performance of the various processing chains.

Other data sources such as ground-based observatories for the period were employed to act as a (semi-) independent check on the accuracy of some of the models. In the following subsections we describe the methods used to validate each product type. A selection of diagnostic output images from the Validation chain are shown in Figs. 3, 4 and 5 .

\subsection{Euler angles}

The Euler angles describe the orientation of the vector magnetometer frame (VFM) to the spacecraft reference frame (CRF) derived from the measurements of the star cameras. The Euler angles are co-estimated with the core field models. It is critical to estimate these angles correctly, as uncertainties in the alignment of the optical bench instruments on the satellite may cause distortions to be intro- 
Table 2. Level 2 products to be validated before release; The small (i) in the name indicates they are intermediate models, prior to validation.

\begin{tabular}{lll}
\hline \multicolumn{1}{c}{ Product Type } & Product Name & \multicolumn{1}{c}{ Chain } \\
\hline Euler Angles & MSW_EULi2C & Comprehensive Inversion \\
& MSW_EULi2D & Dedicated Core \\
\hline Core & MCO_SHAi2C & Comprehensive Inversion \\
& MCO_SHAi2D & Dedicated Core \\
\hline Lithospheric & MLI_SHAi2C & Comprehensive Inversion \\
& MLI_SHAi2D & Dedicated Lithosphere \\
& MLI_SHAi2E & Dedicated Lithosphere (Extended) \\
\hline Ionosphere & MIO_SHAi2C & Comprehensive Inversion \\
& MIO_SHAi2D & Dedicated Ionosphere \\
\hline Magnetosphere & MMA_EULi2C & Comprehensive Inversion \\
\hline Mantle Conductivity & MIN_1DMi2 & 1D Mantle Induction \\
& MIN_3DMi2a & 3D Mantle Induction (Frequency Domain) \\
& MIN_3DMi2b & 3D Mantle Induction (Time Domain) \\
& MCR_1DMi2_ & 1D Mantle C-Response \\
& MCR_3DMi2_ & 3D Mantle C-Response \\
\hline
\end{tabular}

duced, affecting the accuracy of the vector magnetic field values.

Two Euler angle models are computed (a) from the Comprehensive Inversion (MSW_EULi2C) and (b) from the Dedicated Core field model (MSW_EULi2D). Post-launch, there is no independent means to check if the computed Euler angles are correct, so the angles deduced from both models are cross-checked. Small angle rotations can occur while the satellites are in-flight (e.g. from thermal effects) so there may be temporal changes over the mission lifetime. Figure 3(c) shows the timeseries of the estimated mis-alignment between the two reference frames as computed from the CI chain and the Dedicated chain for each Swarm satellite.

All plots and statistics are reported in the final validation report (the MSW_VAL_2_product).

\subsection{Core field}

The core field products define the large-scale slowly varying magnetic field generated within the Earth's outer core. Two core field models are derived from the Comprehensive Inversion chain (MCO_SHAi2C) and the Dedicated Core field chain (MCO_SHAi2D). The models consist of Gauss coefficients of the main field up to spherical harmonic degree 20 , given as a series of snapshots in time. The field models are derived in a complementary fashion: for example, the Comprehensive chain attempts to use as much satellite data as possible (Sabaka et al., 2013), while the Dedicated chain uses quiet time night-side data to minimise the effects of ionospheric fields (Rother et al., 2013).

The validation tests consist of a number of cross-checks between the Swarm core models and other auxiliary models such as the IGRF-11 (Finlay et al., 2010). The largest differences between the independent core models and the Swarm models will be due to the uncertainties in the secular variation prediction, so data from ground-based observatories will also be used to verify the Swarm models. Standard methods of cross checking the core field models include Mauersberger-Lowes specta plots (Fig. 3(a)), degree correlation plots (Fig. 3(b)), and time variations comparison of Gauss coefficients, as well as comparisons to ground observatory data (not shown).

All plots and statistics are reported in the final validation reports (the $\mathrm{MCO} \_\mathrm{VAL} \_2 \mathrm{C}$ and $\mathrm{MCO} \mathrm{VAL}_{-} \_2 \mathrm{D}$ products).

\subsection{Lithospheric field}

The lithospheric field products describe the longwavelength magnetic field generated by the Earth's crust. Three field models are derived: one from the Comprehensive Inversion chain (MLI_SHAi2C) and two from the Dedicated Lithospheric field chain (MLI_SHAi2D and MLI_SHAi2E). The i2C and i2D models describe the field as a spherical harmonic expansion of Gauss coefficients initially from degree and order $L=16-133$, though it is expected that the degree resolution will be extended to $L=150$ or greater as the mission progresses. The $\mathrm{i} 2 \mathrm{E}$ model (i.e. Extended) will be released at the end of the nominal mission as a map of the lithospheric field with a spatial resolution of 30 arc-seconds (Thébault et al., 2013).

The validation tests consist of a number of cross-checks between the Swarm lithospheric models and other auxiliary models such as the MF7 (Maus et al., 2008). The largest differences between the independent lithospheric models and the Swarm models will be due to the uncertainties at higher degrees ( $L>80$, say). Standard methods of cross checking the Swarm models include Mauersberger-Lowes specta plots, degree correlation plots and comparison of Gauss coefficients (examples shown in Figs. 4(a) and (b)).

All plots and statistics are reported in the final validation reports (the MLI_VAL_2C, MLI_VAL_2D and MLI_VAL_2E products).

\subsection{Ionospheric field}

The ionospheric products describe the periodically varying magnetic field generated in the upper atmosphere $(\sim 100-1000 \mathrm{~km})$. They consist of the primary and induced magnetic fields. There are two field models, the first derived from the Comprehensive Inversion chain (MIO_SHAi2C) and the second from the Dedicated Ionospheric field chain (MIO_SHAi2D). The models describe the time-varying ionospheric field for degree and order $L=1-60$ with daily and seasonal temporal variation, and with amplitude linked to the F10.7 solar flux density. The Dedicated model is only 
(a)

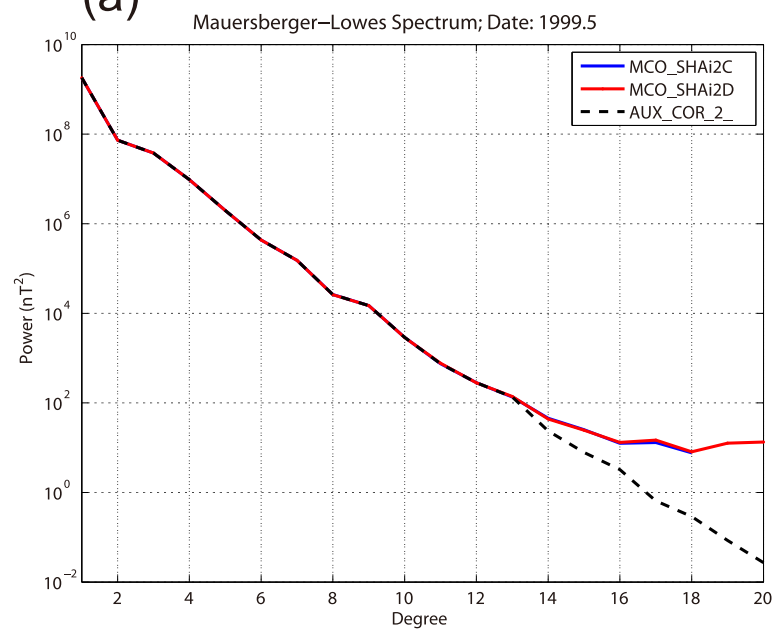

(c)
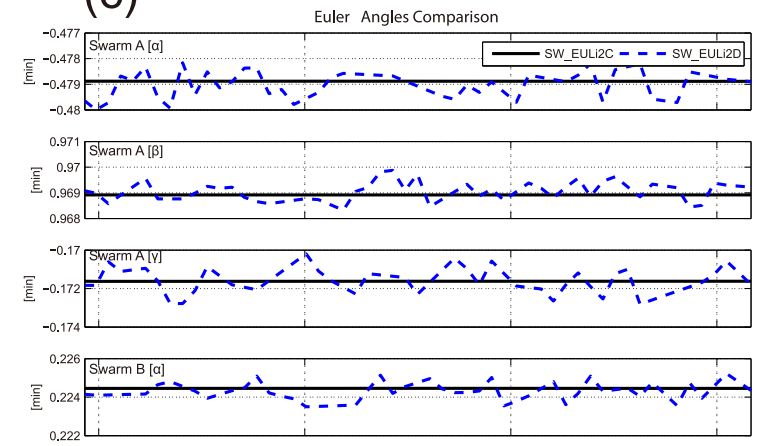

每
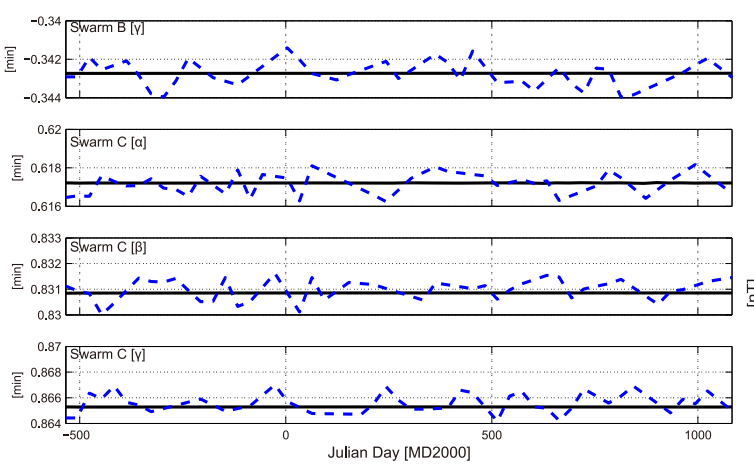

(b)

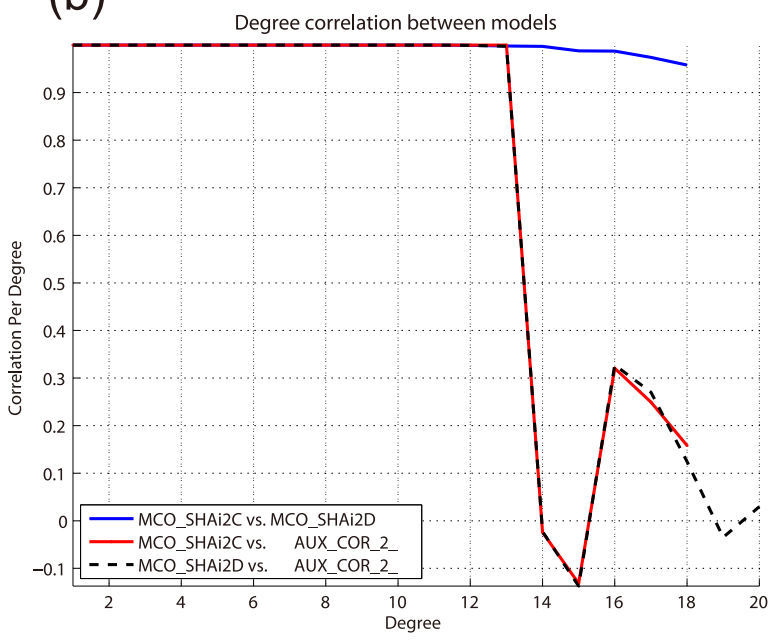

(d)
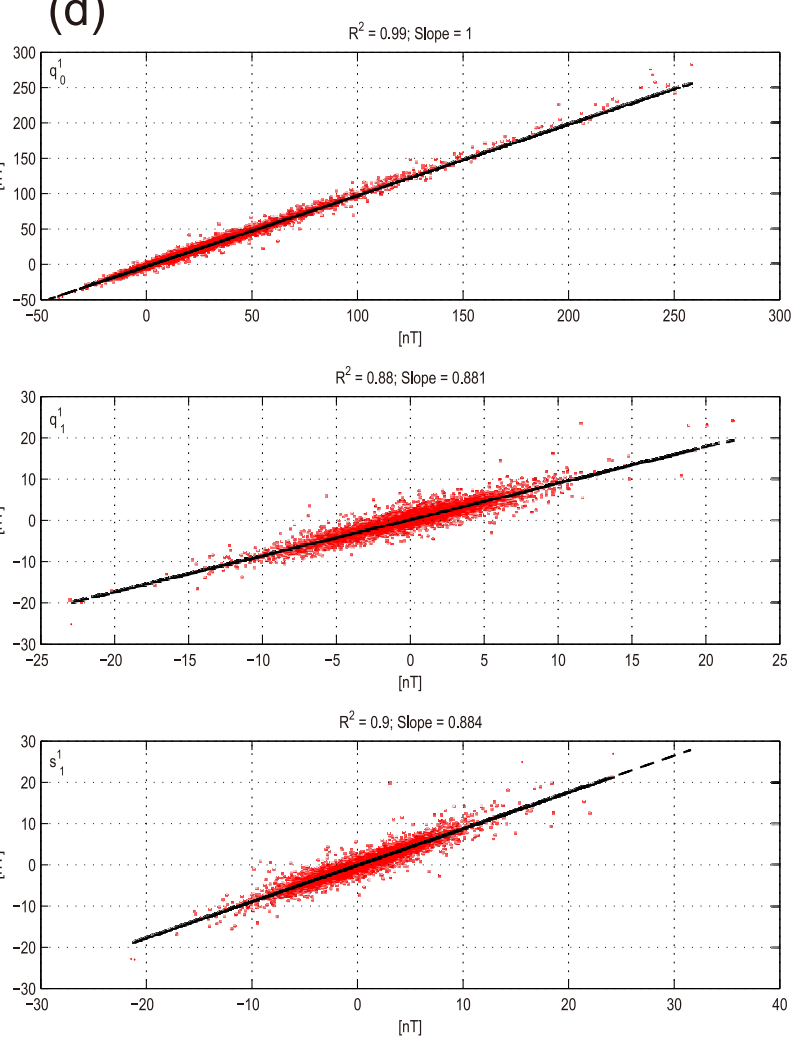

Fig. 3. Example of diagnostic output images from the Validation chain. (a) Mauersberger-Lowes spectra of the Gauss coefficients of the core field models; (b) degree correlation of the Gauss coefficients of the core field models; (c) Euler angle timeseries for each satellite (over the modelled four year lifetime of the mission); (d) correlation of the external dipole Gauss coefficients of the Comprehensive Inversion and 'Fast-track' magnetospheric models.

valid outside the auroral zones $\left( \pm 55^{\circ}\right.$ geomagnetic latitude) (Chulliat et al., 2013), while the CI model attempts to describe the field globally.

The validation tests consist of a number of cross-checks between the Swarm models. There are few recent models of the ionosphere, so data from ground-based observatories will be used to verify the Swarm models, by removing the core, lithosphere and magnetosphere contributions from the hourly mean values (Macmillan and Olsen, 2013). Cross checking the Swarm models involves examining the spatial and temporal variations of the models and maps of the global variation over magnetic local times (e.g. Figs. 4(c), (d), (e)). In addition, plots of comparisons to ground-based observatory data will be produced.

All plots and statistics are reported in the final validation reports (the MIO_VAL_2C and MIO_VAL_2D products).

\subsection{Magnetospheric field}

The magnetospheric product models the large-scale rapidly varying magnetic field generated in the magnetosphere, mostly from the ring current. Only one model, derived from the Comprehensive Inversion chain (MMA_SHAi2C) will be validated, though a second 'fast- 
(a)

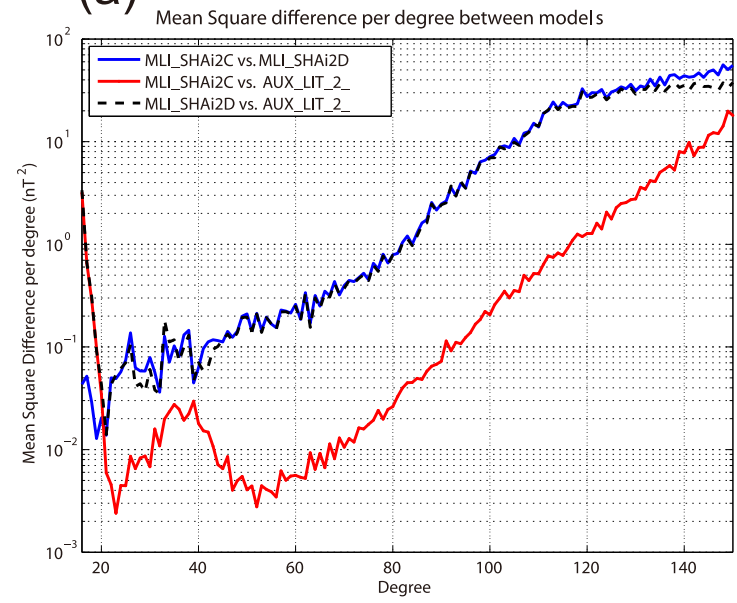

(b)

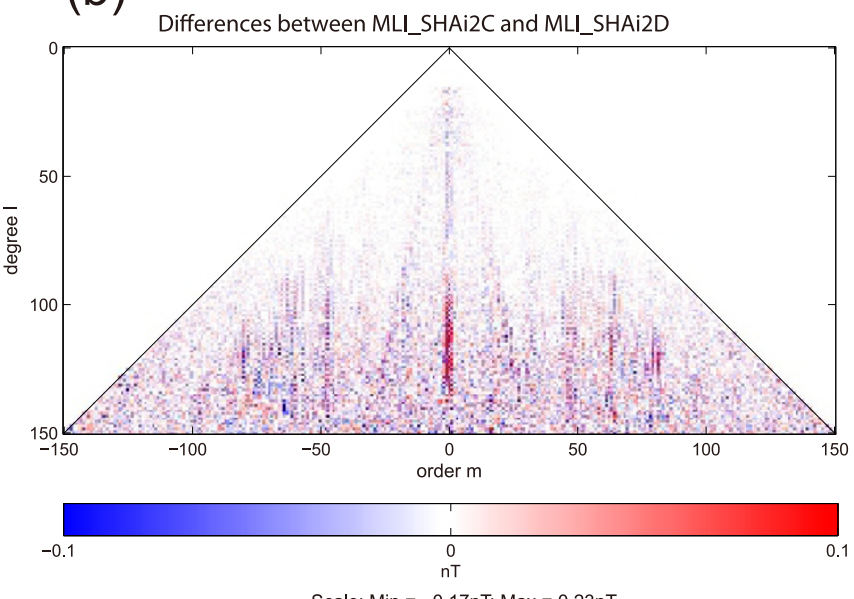

(C) $\quad B_{r}$ Primary
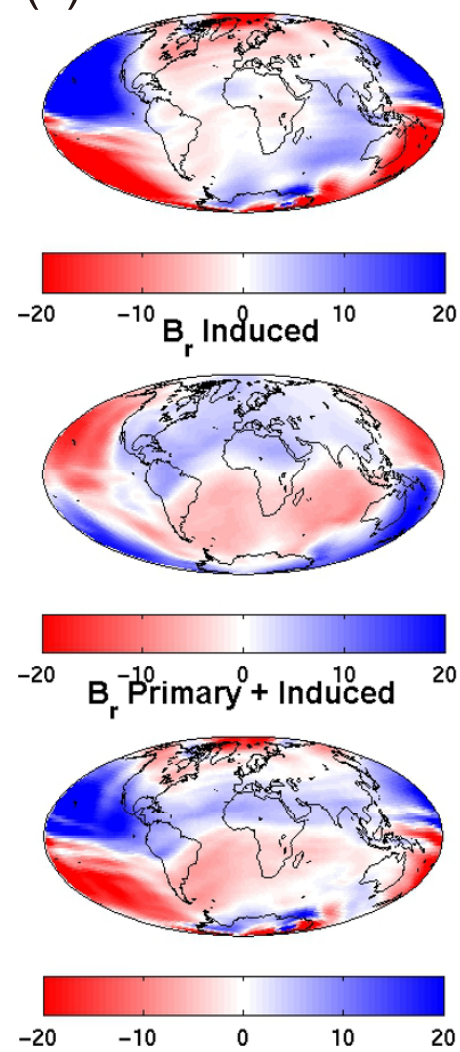

(d)
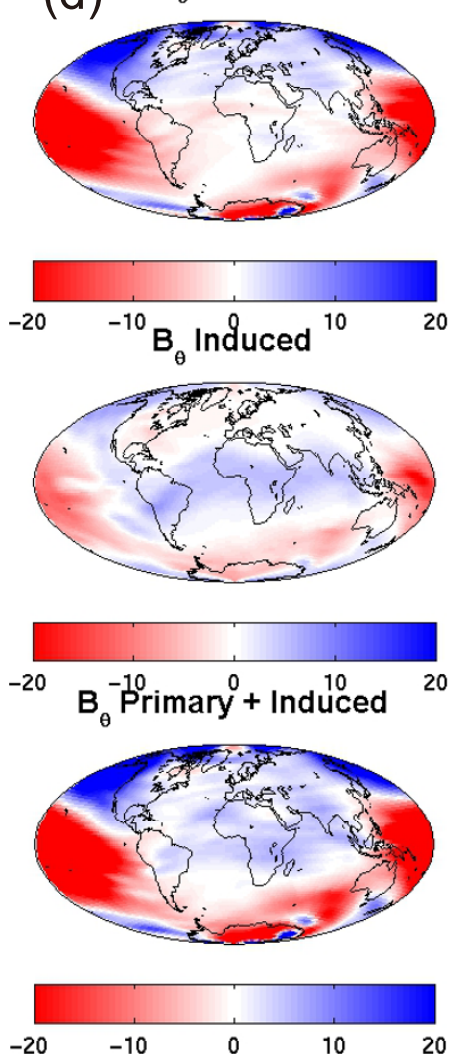

(e)
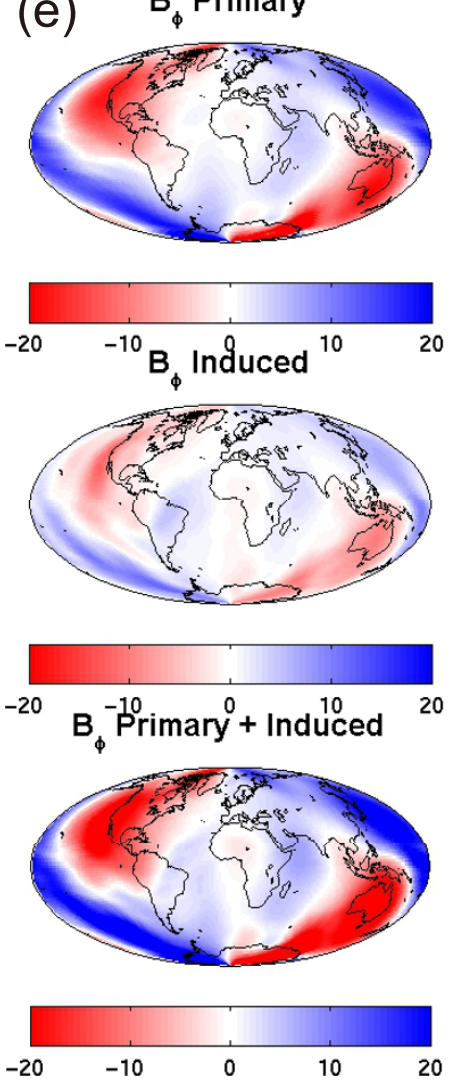

Fig. 4. Example of diagnostic output images from the Validation chain (continued). (a) mean-square differences per degree of the Gauss coefficients for the lithospheric field models; (b) triangle plot visualisation of differences between the Gauss coefficients of the lithospheric field models; (c, d, e) spatial models of the $B_{r}, B_{\theta}$ and $B_{\phi}$ components of ionospheric field at 00:00 Magnetic Local Time on Julian Day -355.

track' model (MMA_SHA_2F) is issued on a daily basis (Hamilton, 2013). The CI model describes the time-varying (external) magnetospheric field up to degree $L=3$ and order $m=1$ with a cadence of ninety minutes for the dipole terms and six hours for the non-dipole terms (Sabaka et al., 2013). A model of the induced (internal) magnetic field up to degree and order $L=5$ will also be produced, again with a cadence of ninety minutes for the dipole terms and six hours for the non-dipole terms.

The validation tests consist of a number of cross-checks between the MMA_SHAi2C and MMA_SHA_2F Swarm models, the Dst indices and data from ground-based observatories after the core, lithosphere and ionosphere contributions have been removed. Cross checking the Swarm models involves examining the correlation of the model Gauss coefficients (e.g. Fig. 3(d)) and with the decomposed Dst (i.e. Est/Ist) indices, which are derived from ground-based observatories.

All plots and statistics are reported in the final validation report (the MMA_VAL_2C product). 
(a)

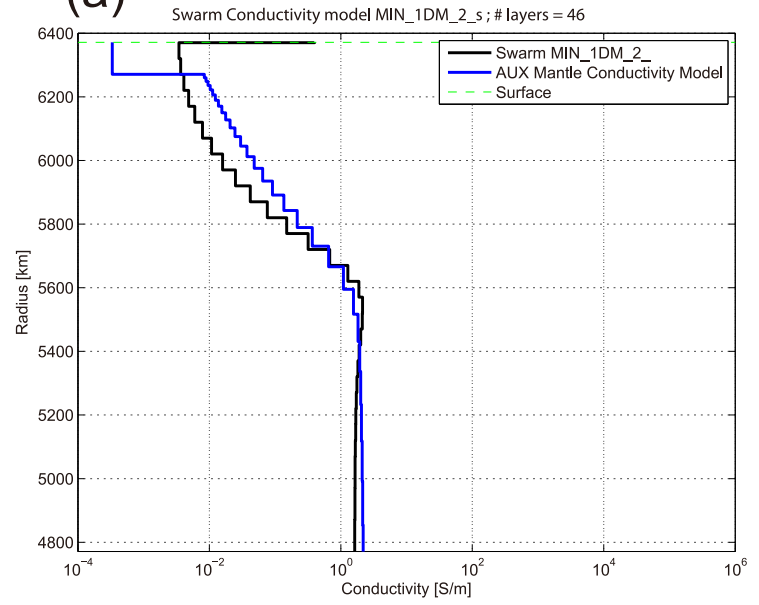

(c)

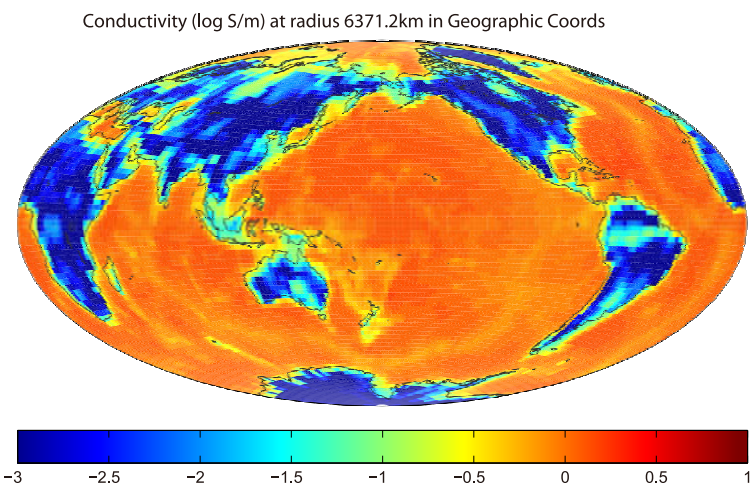

(b)
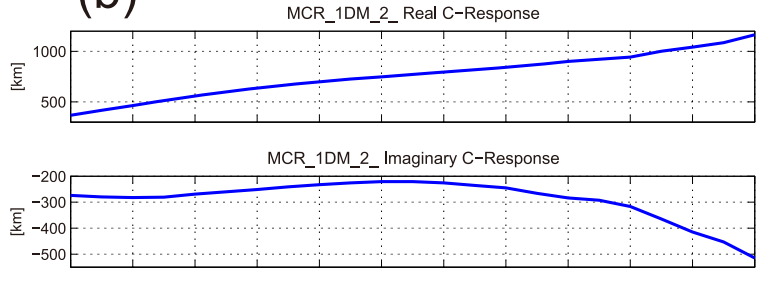

MCR_1DM_2_Error

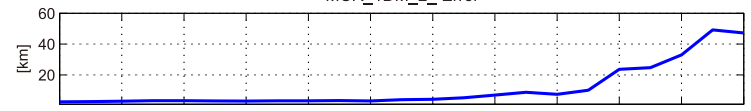

MCR 1DM 2 Coherence

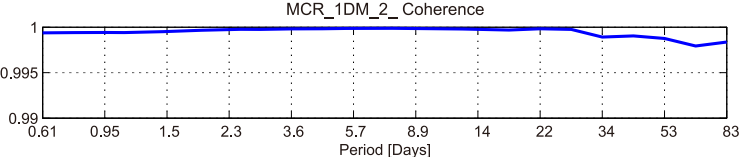

(d)

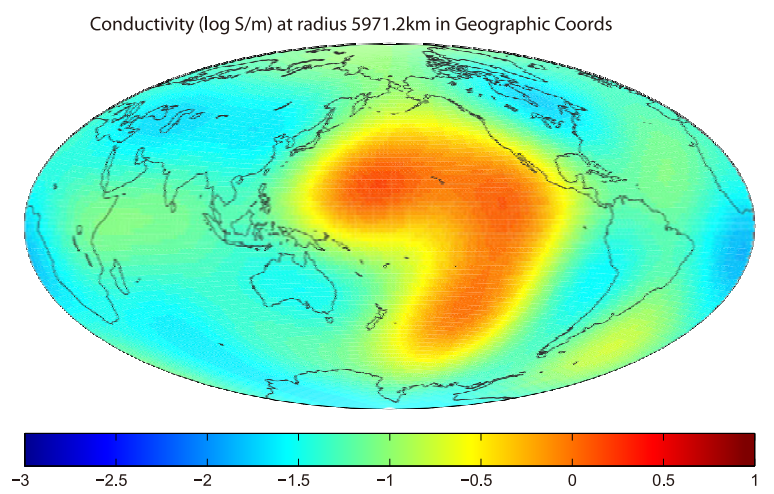

Fig. 5. Example of diagnostic output images from the Validation chain (continued). (a) 1D models of mantle conductivity and (b) 1D model of mantle C-Response induction; (c) 3D conductivity model from the Time Domain approach at the Earth's surface; (d) 3D model of mantle conductivity from the Time Domain approach at a depth of $400 \mathrm{~km}$ showing the recovered image of a synthetic anomaly beneath the Pacific Ocean.

\subsection{Mantle induction and conductivity}

The conductivity of the Earth's mantle is a difficult physical parameter to correctly image. With the additional data from three satellites available, it should be possible to produce relatively detailed $1 \mathrm{D}$ and $3 \mathrm{D}$ models of the mantle conductivity and induction. The products are the MIN_1DMi2_, MCR_1DMi2,, MIN_3DMi2a, MIN_3DMi2b and MCR_3DMi2_ models (Püthe and Kuvshinov, 2013a, b; Veílimský, 2013). The mantle induction and conductivity models require long time period datasets and so will be issued at the end of the nominal four-year mission. A few global 1-D mantle conductivity models exist (e.g. Kuvshinov and Olsen, 2006), which will be compared with the Swarm models (Figs. 5(a) and (b)). However, these are novel products so the validation chain will mostly act as a secondary checking process, for example, plotting out the results in map form (Figs. 5(c) and (d)).

\section{Conclusion}

The Quick Look and Validation chains of the Swarm L2PS provide independent scientific scrutiny of Swarm Level $1 \mathrm{~b}$ and Level 2 products. This evaluation process has already proved its worth. For example, during the test phase of the L2PS the independent validation found a number of inconsistencies between the field model streams, allowing the institutes producing the models to examine and therefore improve their processes and algorithms. Note, the figures shown in this paper are derived from the TDS-1 synthetic dataset and are not meant to be illustrative of the final products.

The Quick-Look and Validation reports will be made available to the general community along with the Level $1 \mathrm{~b}$ and Level 2 products.

Acknowledgments. We thank Alexey Kushinov, Vincent Lesur and a third anonymous reviewer for their comments on improving the draft manuscript. This paper is published with the permission of the Executive Director of the British Geological Survey (NERC).

\section{References}

Chulliat, A., P. Vigneron, E. Thébault, O. Sirol, and G. Hulot, Swarm SCARF Dedicated Ionospheric Field Inversion chain, Earth Planets Space, 65, this issue, 1271-1283, 2013.

Finlay, C. C., S. Maus, C. D. Beggan, T. N. Bondar, A. Chambodut, T. A. Chernova, A. Chulliat, V. P. Golovkov, B. Hamilton, M. Hamoudi, R. Holme, G. Hulot, W. Kuang, B. Langlais, V. Lesur, F. J. Lowes, H. Luhr, S. Macmillan, M. Mandea, S. McLean, C. Manoj, M. Menvielle, I. Michaelis, N. Olsen, J. Rauberg, M. Rother, T. J. Sabaka, A. Tangborn, L. Toffner-Clausen, E. Thébault, A. W. P. Thomson, I. Wardinski, Z. Wei, and T. I. Zvereva, International Geomagnetic Reference Field: the eleventh generation, Geophys. J. Int., 183, 1216-1230, 2010.

Friis-Christensen, E., H. Lühr, and G. Hulot, Swarm: A constellation to 
study the Earth's magnetic field, Earth Planets Space, 58, 351-358, 2006.

Hamilton, B., Rapid modelling of the large-scale magnetospheric field from Swarm satellite data, Earth Planets Space, 65, this issue, 12951308, 2013.

Kuvshinov, A. and N. Olsen, A global model of mantle conductivity derived from 5 years of CHAMP, Ørsted, and SAC-C magnetic data, Geophys. Res. Lett., 33, L18301, 2006.

Macmillan, S. and N. Olsen, Observatory data and the Swarm mission, Earth Planets Space, 65, this issue, 1355-1362, 2013.

Maus, S., F. Yin, H. Lühr, C. Manoj, M. Rother, J. Rauberg, I. Michaelis, C. Stolle, and R. D. Müller, Resolution of direction of oceanic magnetic lineations by the sixth-generation lithospheric magnetic field model from CHAMP satellite magnetic measurements, Geochem. Geophys. Geosyst., 9, Q07021, 2008.

Olsen, N., E. Friis-Christensen, R. Floberghagen, P. Alken, C. D Beggan, A. Chulliat, E. Doornbos, J. T. da Encarnação, B. Hamilton, G. Hulot, J. van den IJssel, A. Kuvshinov, V. Lesur, H. Lühr, S. Macmillan, S. Maus, M. Noja, P. E. H. Olsen, J. Park, G. Plank, C. Püthe, J. Rauberg, P. Ritter, M. Rother, T. J. Sabaka, R. Schachtschneider, O. Sirol, C. Stolle, E. Thébault, A. W. P. Thomson, L. Tøffner-Clausen, J. Velímský, P. Vigneron, and P. N. Visser, The Swarm Satellite Constellation Application and Research Facility (SCARF) and Swarm data products, Earth Planets Space, 65, this issue, 1189-1200, 2013.

Püthe, C. and A. Kuvshinov, Determination of the 1-D distribution of electrical conductivity in Earth's mantle from Swarm satellite data, Earth Planets Space, 65, this issue, 1233-1237, 2013a.
Püthe, C. and A. Kuvshinov, Determination of the 3-D distribution of electrical conductivity in Earth's mantle from Swarm satellite data: Frequency domain approach based on inversion of induced coefficients, Earth Planets Space, 65, this issue, 1247-1256, 2013b.

Rother, M., V. Lesur, and R. Schachtschneider, An algorithm for deriving core magnetic field models from the Swarm data set, Earth Planets Space, 65, this issue, 1223-1231, 2013.

Sabaka, T. and N. Olsen, Enhancing comprehensive inversions using the Swarm constellation, Earth Planet Space, 58, 371-395, 2006.

Sabaka, T. J., L. Tøffner-Clausen, and N. Olsen, Use of the Comprehensive Inversion method for Swarm satellite data analysis, Earth Planets Space, 65, this issue, 1201-1222, 2013.

Thébault, E., P. Vigneron, S. Maus, A. Chulliat, O. Sirol, and G. Hulot, Swarm SCARF Dedicated Lithospheric Field Inversion chain, Earth Planets Space, 65, this issue, 1257-1270, 2013.

Velímský, J., Determination of three-dimensional distribution of electrical conductivity in the Earth's mantle from Swarm satellite data: Timedomain approach, Earth Planets Space, 65, this issue, 1239-1246, 2013.

Visser, P., E. Doornbos, J. van den IJssel, and J. T. da Encarnação, Thermospheric density and wind retrieval from Swarm observations, Earth Planets Space, 65, this issue, 1319-1331, 2013.

C. D. Beggan (e-mail: ciar@bgs.ac.uk), S. Macmillan, B. Hamilton, and A. W. P. Thomson 